\section{Jahrestagung Leipzig}

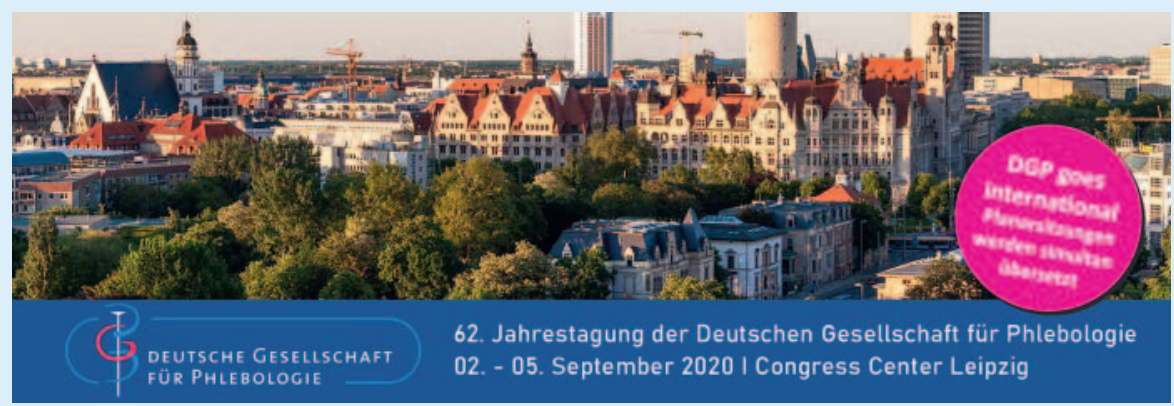

Die Jahrestagung der DGP findet vom 2.-5.9.2020 unter dem Motto „Gemeinsam in Leipzig - Phlebologie international und national“ in Leipzig statt.

Das Programm, das von unserem Kongresspräsidenten Dr. Tobias Hirsch vorbereitet wurde, können Sie unter www.phlebologie2020.de jederzeit mit den jeweiligen Aktualisierungen abrufen.

Auch in diesem Jahr verleiht die DGP wieder in Kooperation mit Partnern aus der Industrie traditionell im Rahmen der Jahrestagung verschiedene Wissenschaftspreise. Dies sind im Einzelnen:

\section{Redner- und Posterpreise}

2 Preise à 500 Euro für die besten Präsentationen im Rahmen der Jahrestagung. Die Preisträger für diese Preise werden im Rah- men des Kongresses von einer Jury ausgewählt.

\section{Bauerfeind-Doktorandenpreis}

Der Doktorandenpreis richtet sich an junge Mediziner mit wissenschaftlichem Interesse auf den Gebieten Phlebologie, Lymphologie und verwandten Disziplinen.

Bevorzugt werden Arbeiten, welche die Kompressionstherapie in die Untersuchungen einbeziehen. Ein Preisgeld in Höhe von 5000 Euro pro Preisträger wird vergeben (1500 Euro bei der Auszeichnung seines Projekts und weitere 1500 Euro bei Vorlage seiner Dissertationsschrift; die verbleibenden 2000 Euro gehen an den Erstbetreuer und die dahinterstehende Institution). Bewerbungen und nähere Informationen bis zum 10.7.2020 über medwiss@bauerfeind.com (z. Hd. Dr. Antje Mark).

\section{Kreussler Young Phlebologists' Travel} Award

Kreussler Pharma verleiht anlässlich der Jahrestagung der Deutschen Gesellschaft für Phlebologie unter allen eingereichten Abstracts 2 Travel Awards in Höhe von je 1000 Euro. Der Award wird regelmäßig im Rahmen dieses Kongresses zur Förderung des wissenschaftlichen Nachwuchses vergeben. Das mit jeweils 1000 Euro dotierte Reisestipendium soll Sie bei der Teilnahme am Kongress und der Präsentation Ihrer wissenschaftlichen Arbeit unterstützen.

medi Reise- und Teilnahmestipendien für Arzt-Berufsstarter

medi unterstützt interessierte Ärztinnen und Ärzte, die am Anfang ihrer beruflichen Laufbahn stehen, mit Reise- und Teilnahmestipendien. Die Reise- und Teilnahmestipendien umfassen den freien Eintritt an allen Kongresstagen sowie die Fahrt- und Hotelkosten bis zu einem Betrag von 500 Euro. Bewerbungsfrist ist der 15.7.2020. Der Bewerbungsbogen und weitere Informationen können angefordert werden bei: medi, Frau Strauss, a.strauss@medi.de.

\section{Sigvaris-Förderpreis Phlebologie}

Der Sigvaris-Förderpreis Phlebologie richtet sich an junge Wissenschaftler und Mediziner. Die Bewerber sollten Interesse an wissenschaftlicher Arbeit und Spaß an aktueller 
Berichterstattung haben. Die Verleihung erfolgt auf der Jahrestagung. Sie können Ihre Bewerbung mit einem aussagefähigen Lebenslauf bis zum 15.7.2020 einreichen bei: cordula.berger@sigvaris.com.

\section{Juzo-Forschungspreis Phlebologie}

Der Juzo-Forschungspreis Phlebologie wird in Kooperation mit der Deutschen Gesellschaft für Phlebologie vergeben. Er soll die Wissenschaft in der interdisziplinären Kompressionstherapie unterstützen und wird vergeben an Mediziner mit wissenschaftlichem Interesse auf den Gebieten Phlebologie, Lymphologie, Dermatologie und verwandten Disziplinen. Gefördert werden Projektkonzepte, welche explizit die Kompressionstherapie in die Untersuchungen einbeziehen. Der Preis ist mit 5000 Euro ausgeschrieben. Der Preis wurde im Rahmen der Jahrestagung der Deutschen Gesellschaft für Phlebologie erstmals 2019 in Münster vergeben. Bewerbungen richten Sie bitte bis zum 15.7.2020 an das Sekretariat der Deutschen Gesellschaft für Phlebologie: Anja.Pielhau@ukbonn.de.

\section{Zoltan-Varady-Vortragspreis}

Herr Prof. Dr. Zoltan Varady stiftet in Kooperation mit der Deutschen Gesellschaft für Phlebologie einen Wissenschaftspreis im Rahmen unserer Jahrestagung. Jeweils der beste Vortrag im Bereich der Minichirurgie oder Mikrochirurgie wird mit einem Vortragspreis von 1000 Euro prämiert. Die Preisjury besteht aus 3 Mitgliedern der VOP-AG.

\section{DGP International Educational Grants}

Die DGP verleiht in Kooperation mit der Industrie International Educational Grants, die die Teilnahme an der International UItrasound Master Class, den Kongresseintritt am 4. und 5.9.2020 und den Festabendeintritt beinhalten. Wenn Sie PhD-Student oder "Jung-Phlebologe“ und nicht älter als 39 Jahre sind, bewerben Sie sich bitte bis zum 1.7.2020 über die Kongresswebsite: https://phlebologie-2020.de/informationenglish/dgp-international-educationalgrant/.

Am 4.9.2020 findet das Update Wundmanagement für die Pflegeberufe statt mit einer ausgewogenen Mischung aus Theorie und Praxis. Die ICW-Rezertifizierung mit 8 Fortbildungspunkten ist bereits genehmigt, sodass Ihre Mitarbeiter allein mit dieser 1-tägigen Veranstaltung ihrer Rezertifizierungspflicht nachkommen können. Nähere Informationen erhalten Sie über: https://phlebologie-2020.de/update_wund management/.

Wir würden uns freuen, auch Ihre Mitarbeiter in Leipzig begrüßen zu können.

Am Donnerstag, dem 3.9.2020, wird von 9:00-18:00 Uhr ein Ultrasound Masterclass Course unter der Leitung von Frau Dr. Mendoza stattfinden. Das Programm sieht Folgendes vor:

\section{Ultrasound Masterclass $\mid 3^{\text {rd }}$ September 2020, 9am-6pm \\ Masterclass on Duplex Ultrasound of Superficial Leg Veins}

by Erika Mendoza, MD, PhD|Language: English

\begin{tabular}{|c|c|}
\hline Time & Masterclass Duplex \\
\hline 09:00 & Welcome and Presentation \\
\hline 09:15 & $\begin{array}{l}\text { Basics: How, where, whom to inves- } \\
\text { tigate? Flow and Reflux, Physiology, } \\
\text { Provocation maneuvers }\end{array}$ \\
\hline $10: 00$ & $\begin{array}{l}\text { Great saphenous vein, sapheno- } \\
\text { femoral junction Valves, Flow } \\
\text { patterns }\end{array}$ \\
\hline $11: 00$ & Coffee break \\
\hline $11: 15$ & Live Patient demonstration \\
\hline $12: 00$ & $\begin{array}{l}\text { Short saphenous vein, Perforating } \\
\text { veins }\end{array}$ \\
\hline $12: 45$ & Lunch break \\
\hline $13: 30$ & Live patient demonstration \\
\hline $14: 15$ & $\begin{array}{l}\text { Superficial vein thrombosis, } \\
\text { findings "on the way" }\end{array}$ \\
\hline $15: 00$ & Coffee break \\
\hline $15: 45$ & $\begin{array}{l}\text { Decision how to treat depending on } \\
\text { the findings }\end{array}$ \\
\hline $16: 30$ & Life Patient demonstration \\
\hline $17: 15$ & $\begin{array}{l}\text { Postoperative findings, complica- } \\
\text { tions, follow-up }\end{array}$ \\
\hline
\end{tabular}

IMPRESSUM

Verantwortlich für Mitteilungen der DGP:

Dr. med. Erika Mendoza 